\title{
Making MRI available for patients with cardiac implantable electronic devices: growing need and barriers to change
}

\author{
A. N. Bhuva ${ }^{1,2}$ (D) R. Moralee ${ }^{1}$ J. C. Moon ${ }^{1,2} \cdot$ C. H. Manisty ${ }^{1,2}$
}

Received: 3 July 2019 /Revised: 2 August 2019 / Accepted: 12 September 2019/Published online: 27 November 2019

(C) The Author(s) 2019

\begin{abstract}
More than half of us will need a magnetic resonance imaging (MRI) scan in our lifetimes. MRI is an unmatched diagnostic test for an expanding range of indications including neurological and musculoskeletal disorders, cancer diagnosis, and treatment planning. Unfortunately, patients with cardiac pacemakers or defibrillators have historically been prevented from having MRI because of safety concerns. This results in delayed diagnoses, more invasive investigations, and increased cost. Major developments have addressed this - newer devices are designed to be safe in MRI machines under specific conditions, and older legacy devices can be scanned provided strict protocols are followed. This service however remains difficult to deliver sustainably worldwide: MRI provision remains grossly inadequate because patients are less likely to be referred, and face difficulties accessing services even when referred. Barriers still exist but are no longer technical. These include logistical hurdles (poor cardiology and radiology interaction at physician and technician levels), financial incentives (re-imbursement is either absent or fails to acknowledge the complexity), and education (physicians self-censor MRI requests). This article therefore highlights the recent changes in the clinical, logistical, and regulatory landscape. The aim of the article is to enable and encourage healthcare providers and local champions to build MRI services urgently for cardiac device patients, so that they may benefit from the same access to MRI as everyone else.

Key Points

- There is now considerable evidence that MRI can be provided safely to patients with cardiac implantable electronic devices (CIEDs). However, the volume of MRI scans delivered to patients with CIEDs is fifty times lower than that of the estimated need, and patients are approximately fifty times less likely to be referred.

- Because scans for this patient group are frequently for cancer diagnosis and treatment planning, MRI services need to develop rapidly, but the barriers are no longer technical.

- New services face logistical, educational, and financial hurdles which can be addressed effectively to establish a sustainable service at scale.
\end{abstract}

Keywords Pacemaker, artificial · Defibrillators, implantable $\cdot$ Magnetic resonance imaging

\section{Abbreviations \\ CIED Cardiac implantable electronic devices \\ ICD Implantable cardioverter-defibrillator}

MRI Magnetic resonance imaging

PPM Permanent pacemaker

SAR Specific absorption rate
A. N. Bhuva

anish.bhuva@nhs.net

1 Department of Cardiac Imaging, Barts Heart Centre, Barts Health NHS Trust, West Smithfield, London EC1A 7BE, UK

2 Institute for Cardiovascular Science, University College London, London, UK

\section{Introduction}

Magnetic resonance imaging (MRI) is fundamental to healthcare, particularly for cancer diagnosis and treatment (surgery, radiotherapy), for diseases of the central nervous and musculoskeletal systems. Sixty million scans are 
performed annually worldwide [1], and it is the fastest growing imaging modality at $12 \%$ annually (Fig. 1) [2]. MRI is beginning to replace invasive biopsy for diagnosis of some cancers and is now often essential for planning of neurosurgical procedures and CyberKnife radiotherapy [4].

However, one in 50 over the age of 75 has a permanent pacemaker (PPM) or implantable cardioverter-defibrillator (ICD) - these are collectively termed cardiac implantable electronic devices (CIEDs) [3, 5]. Historically, MRI has been contraindicated for safety but each of these individuals has an estimated need for MRI in their lifetime of 50-75\%, particularly because they are older and have more comorbidities [6]. Now, technical developments have made it reasonable to perform MRI for these individuals [7], but provision remains grossly inadequate due to logistical difficulties. This review therefore discusses the significant non-technical barriers to developing a sustainable service.

\section{Safety}

Overall, MRI is an extremely safe imaging modality, with over 300 million scans performed worldwide to date $[8,9]$. There have been reported safety events in patients who did not undergo CIED reprogramming prior to an MRI scan [10]. Most likely, this was because radiology teams were not aware of the presence of a CIED, because it was not detected by staff or volunteered by the patient. To the authors' knowledge, there have been no deaths attributed to the performance of MRI in patients with CIEDs when performed intentionally, with the correct protocols followed. In patients with CIEDs, the risk has been attributed to the interaction between magnetic field and device components [11]. Adequate patient monitoring and safety procedures are therefore paramount, and so scanning guidelines for CIED patients have recently been published in the UK, Europe, and the USA [12-16].

\section{New technology}

To address the safety issues, an industry-wide effort was needed to develop MRI-conditional devices [17]. Software modifications with an "MRI mode" were introduced so reprogramming before and after scanning became straightforward and electromagnetic interference minimized. Since their approval in 2008, multiple studies and over 10,000 scans have demonstrated their safety, and are detailed elsewhere [7, 12].

If an MRI-conditional generator is combined with MRIconditional leads from a different manufacturer, however, this renders the system non MRI-conditional because these components have not been tested together. Despite little clinical evidence of risk associated with non MRI-conditional leads undergoing MRI, increased perception of risk leads to difficulties with access for these patients [18].

\section{Non MRI-conditional CIEDs}

The majority of patients with CIEDs currently in situ however still have non MRI-conditional "legacy" devices implanted. The situation is rapidly changing for scanning these devices and professional guidelines are now endorsing scanning if there is a clear indication after an individual risk-benefit assessment $[12,14]$. The evidence base is large with two studies showing that the risk of scanning legacy pacemakers and defibrillators is tiny. Provided strict protocols under close medical supervision were adhered to, there have been no major events in 3600 scans where programming was performed

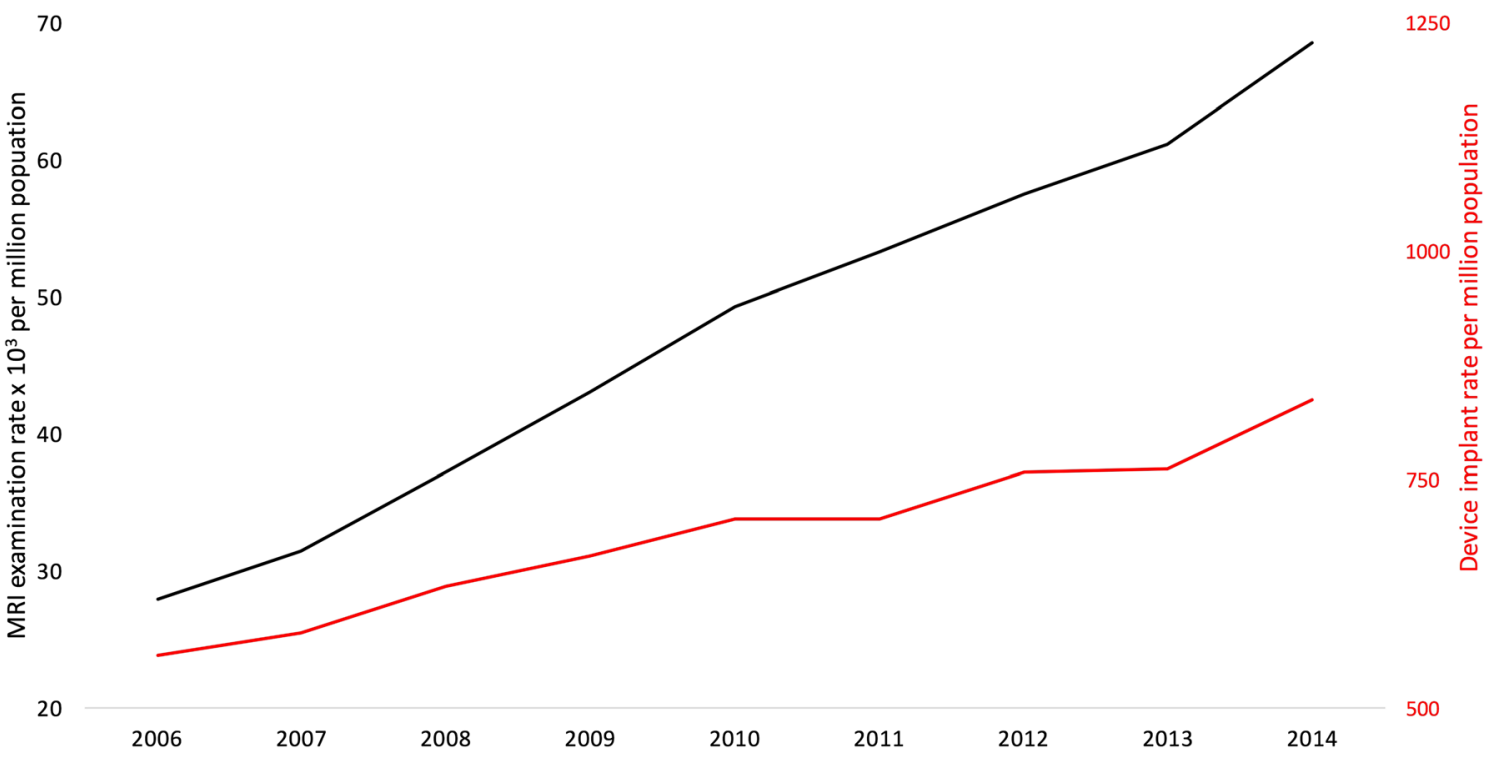

Fig. 1 Annual total device implant rate (red) and MRI scan provision (black) in England [2, 3] 
correctly $[15,16]$. The risk is particularly low when compared with a major complication rate of 0.4 to $2 \%$ associated with elective laser-assisted lead extraction to replace a legacy system with an MRI-conditional CIED [19, 20]. In a prospective registry (the REPLACE registry), the rate of major complications among patients undergoing generator replacement with or without the placement of an additional transvenous lead was 4 to $15 \%$ [21]. It is however difficult to prove that MRI scanning in all circumstances is completely safe - there are an almost infinite number of lead-generator combinations that would require testing. For the patient and healthcare system, the test should be whether it is safer to scan than not to scan. Fortunately, major societies now provide protocols related to MRI scanning in patients with non MRI-conditional devices $[12,14,22]$.

\section{Where are we at the moment?}

Patients with CIEDs and clinicians however have reported severe difficulties accessing MRI scans (even for those with MRI-conditional devices), with requests often declined without clear reason [23]. The scale of the problem is large, but hard to quantify - requests are censored by patients and referrers, as well as by the lack of service provision. US data suggests that patients with CIEDs are between 40 and 50 times less likely to be referred for MRI than they should be, and refusal rates for non-clinical reasons (logistical/financial/ access) remain high $[24,25]$. Similar experience has been reported in Ireland, Italy, and the UK, with $73 \%$ reporting delays in receiving appointments [26-29]. In an Italian prospective questionnaire study, $39 \%$ of patients with CIEDs were denied MRI scans. Fifty percent of whom reported being denied because they had a CIED implanted, even though MRI-conditional [28].

\section{An ignored and growing health inequality}

We start from a low level of provision, and major barriers still exist. A national audit covering $86 \%$ of hospitals in England reported there were less than 1000 scans performed annually and less than half of units will scan MRI-conditional devices [26]. An estimated 50,000 MRI scans are needed a year in the UK for patients with CIEDs, meaning this is a 50 -fold underprovision (Supplementary Data) [2,3]. MRI capacity required is similar to other countries with reported data. Estimates in the USA are for 200,000 scans a year in a patient population four times the size, consistent with prospective European data $[28,30]$. Bridging this gap would expand total MRI activity by around 2\% (current annual growth for MRI services is at $12 \%$ ) [2]; however, it is likely to be offset by reduced activity in other areas.
The need is growing by $10-15 \%$ a year (Fig. 1). Alongside increasing demand for MRI, CIED implantation is rising by $5 \%$ per year. Half of this group is aged over 65 years with increased comorbidities and therefore will rely on optimal diagnostic imaging [5]. Because equipment to perform these scans is available in most MRI departments [26], it should mean that these scans are now provided and the need is met. The focus of the remainder of the review is to understand the remaining barriers; however, it is first necessary to understand the extra logistical safety steps needed that give rise to these (Fig. 2).

\section{Protocols for MRI in patients with CIEDs}

Before the scan, it is necessary to check whether the CIED is indeed MRI-conditional — with both leads and generator

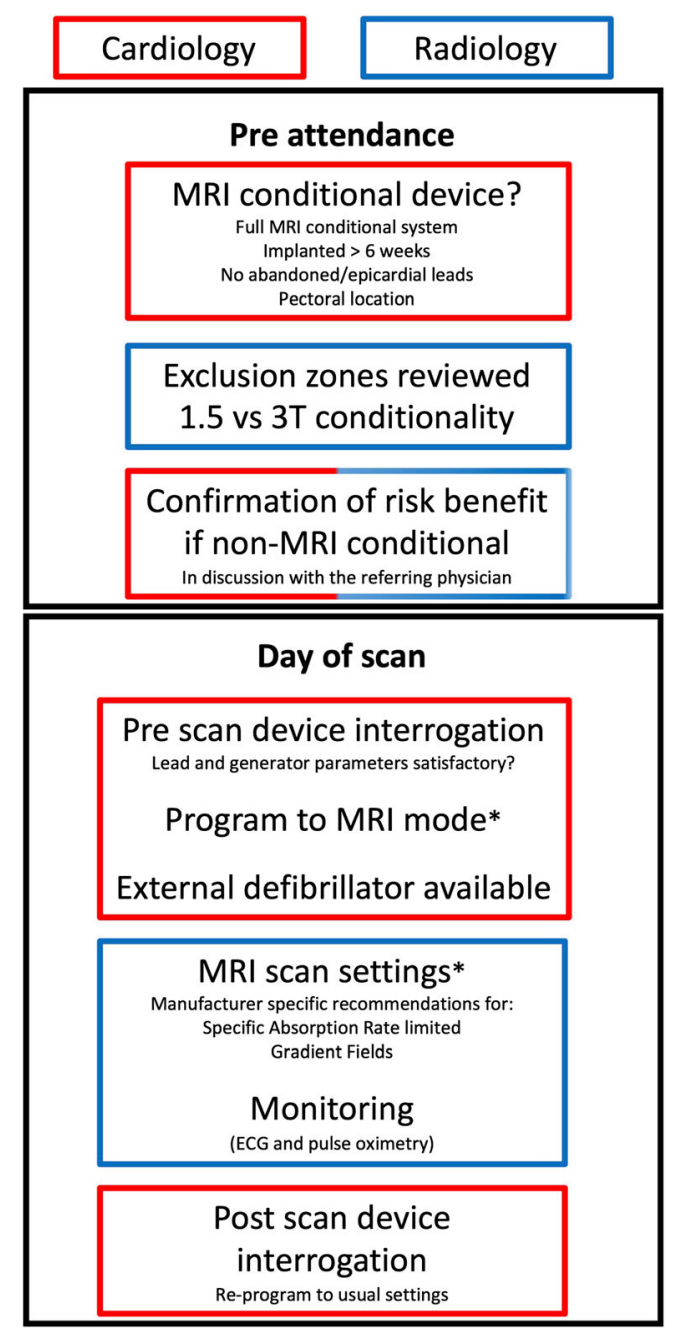

Fig. 2 Logistical steps to provide MRI for patients with CIEDs. Manufacturer-specific guidances are available for MRI-conditional devices available at mrimypacemaker.com. At steps indicated by an asterisk symbol, follow published guidelines for Non MRI-conditional devices $[12,16]$ 
comprising part of an MRI-conditional system. This can be done via referrers, patient records, device identification cards (given to the patient at the time of implantation), or the radioopaque markers on leads and pulse generators seen on chest $\mathrm{x}$ ray. This often presents the greatest obstacle to scanning, as patients have their devices implanted in different clinical facilities to where their MRI is requested. An increasing number of patients now hold details of their device themselves, which can facilitate this process. Some older, previously non-MRIconditional leads and generators have now been retrospectively tested and approved as MRI-conditional. This means that labelling of a device as non MRI-conditional cannot be taken as a definitive, permanent categorization, potentially leading to confusion for clinicians and patients alike. Each device manufacturer offers an MRI device check tool in order to assess the suitability of an individual patient's device components to address this issue, centralized at mrimypacemaker. com. Non MRI-conditional devices require that a clear indication is established through discussions between clinicians and patient, and protocols under close supervision are followed. Increasingly, there are centers that scan almost all patients with CIEDs, meaning that the practice of checking device conditionality prior to scanning can be streamlined.

Before the scan (usually on the same day), the device is checked and programmed using a portable unit by a cardiologist or cardiac physiologist. For patients with MRI-conditional devices, this involves straightforward re-programming to "MRI-Safe" mode, and for those patients with legacy devices, this involves manual re-programming. There is also a safety checklist to identify patients deemed higher risk for undergoing MRI scanning. The presence of an epicardial or abandoned lead may be an exclusion at a particular institution, but this type of patient is not addressed by current recommendations. There is a growing understanding of the risks and risk mitigation in these scenarios, so patients are not systematically excluded [31]. Overly rigid selection protocols may therefore cause patients more harm (from not scanning) than good.

During the scan, the patient should be monitored using both electrocardiography and pulse oximetry. An advanced cardiac life support-trained person should be present for the duration of the scan, regardless of MRI-conditionality [12]. An external defibrillator with pacing capability should be immediately available. On MRI completion, the device must be interrogated and restored to the initial settings - either in the department or scanner side.

\section{MRI scanning conditions}

CIEDs are only MRI-conditional under specific controls of the MRI environment intended to lower power. This includes the region of imaging, field strength, spatial gradients, and specific absorption rate (SAR). Specific conditions can be found in manufacturer device check tools. The use of SAR may be unreliable in the presence of a CIED, and so manufacturers are moving towards reporting a more precise measure of energy deposition, the root mean square value of the B1 field (B1+RMS) [32].

\section{MRI artifact}

Diagnostic imaging can be limited due to metallic artifact, but this is usually restricted to cardio-thoracic scans. Artifact is related to the type and proximity of the device, being more prominent in ICDs than PPMs due to the greater off-resonance induced by the battery and high-voltage transformer. Strategies to reduce artifact generally yield interpretable results and so ensure clinical yield is high, which is particularly important for patients with ICDs [33]. These strategies include using spoiled gradient echo cines, shorter echo times, or wideband inversion recovery pulses for late gadolinium enhancement [34, 35].

\section{An early diagnosis makes clinical and economic sense}

When MRI scans are provided for CIED patients, about a third are for acute indications and another third yield a positive cancer diagnosis [36]. The impact on decision-making is remarkably high $[33,37]$, but is limited to the experience of a few centers [26]. The consequences of not performing these scans can be devastating, particularly for cancer, half of transient ischemic attacks or spinal cord compression - where delayed diagnosis and travelling great distances for an MRI scan are particularly unwarranted [29]. Change must therefore be implemented rapidly.

The benefit is evident both for the patient and healthcare system. Timely scanning is important for all these indications and has a financial benefit in addition to the patient care- - a prompt cancer diagnosis for example saves $\$ 5000$, and an extra inpatient bed-day costs $\$ 300$ [38, 39]. Medical bodies and health economists recognize this need [40], and successive reports have highlighted the clinical and financial costs of a late diagnosis [41].

\section{Barriers to scale and what we need}

Referrers and service providers still perceive safety concerns. One survey assessing current practice attributed this perception to other hurdles including logistical problems, a lack of inter-disciplinary support, poor education, and reimbursement [26]. Reluctance to develop services has been observed in many countries [27]. Information to check device 
compatibility is disparate and requires accessing different manufacturer guidelines and cardiology and radiology protocols. This means centers lack confidence because information is not easily accessible and adds on time to preparation and scanning. Already busy services therefore have valid concerns about managing rising demand. Many patients with legacy devices find it difficult to accept that they can undergo MRI scanning, as they have been told repetitively in the past that they can never undergo this. Re-education is therefore required at every level of an institution-from the patient to the cardiologist, as well as referring physicians/surgeons and radiology departments.

Scanner and device manufacturers are working to break down some of these barriers. The latest pacemakers can be pre-programmed days in advance to anticipate a scan and will sense the MRI environment to switch in and out of MRI-mode when the patient enters and leaves the scan room [42]. This avoids MRI department workflows being disrupted by reliance on cardiac device physiologists being available at the same time as the MRI. Some pacemakers have a portable hand-held activator that can be activated by the patient prescan [42]. While this provides some flexibility, it remains necessary to interrogate devices after MRI to ensure correct function. MRI manufacturers are also introducing software packages to simplify adjustments to scanner settings [43]. Such technological aids are appreciated by clinicians but refining care pathways to minimize the multi-disciplinary workflow represents the larger preceding hurdle. Even when strict protocols are followed, complications are rare, making this a resource-intensive but low-yield risk reduction strategy.

Locally, it has been useful to centralize expertise in a few named individuals, including administration staff, within each department. A standardized and specific referral form that is completed by referrers prior to booking a scan helps to reduce delays. MRI scans for patients with CIEDs have also been redesigned into dedicated lists, whereby all necessary staff are present. This however involves reaching across standard silos of practice in a hospital, but facilitates easy decisionmaking and improving confidence (particularly at the early stages of setting up a service) [29].

\section{Current progress}

It will soon be indefensible not to provide this service, and 2018 represented a year of policy change. The Centers for Medicare and Medicaid Services recently changed funding guidance for non MRI-conditional CIEDs and now states there is sufficient evidence for the coverage of MRI in CIEDs that do not have FDA labelling, unless there is a fractured or abandoned lead [44]. The UK Royal College of Radiology and British Cardiovascular Society released a joint statement recognizing the need to develop more services [45].
The statement acknowledges the devastating consequences of not undergoing MRI and the growing health inequality. This represents high-level consensus to develop new working practices and partnerships and should de-risk hospitals from establishing new services. Legal ambiguity however still remains a problem. As noted by the German Cardiac and Roentgen Societies, apportioning responsibility between radiology and cardiology departments can make establishing interdisciplinary services complex [46]. In Italy, contradicting laws both implicitly allow and explicitly forbid MRI scanning in this setting at the same time [23].

Remarkably, hospitals frequently implant an MRIconditional CIED, spending the extra money but do not offer to scan it. Cardiology standards are now addressing this, beginning to explicitly ask CIED implantation centers to ensure pacing support for MRI units [47]. The 2018 British Heart Rhythm Society Standards for Implantation and Follow-up of Cardiac Rhythm Devices state: "Each device centre must ensure that they have agreements and arrangements in place that allow their patient's access to MRI scanning. Patients should not be denied access to MRI scanning because of lack of these arrangements or resource." Implementing an MRI service for CIED patients is therefore now an imperative for radiology and cardiology practices. An important next step will be to establish billing arrangements that recognize the scan complexity.

\section{Conclusion}

Clinical demand for MRI in patients with these devices is high; however, provision remains poor. Change must now be rapid to resolve this for patients who need diagnosis and treatment planning of otherwise terminal pathology. Addressing this will require education of clinicians, referrers, and patients alongside strategies for streamlining workflows, improving re-imbursement, and developing new collaborations.

Funding information $\mathrm{AB}$ is supported by a doctoral research fellowship from the British Heart Foundation (FS/16/46/32187). JCM and CM are directly and indirectly supported by the University College London Hospitals, NIHR Biomedical Research Centre and Biomedical Research Unit at Barts Hospital, respectively.

\section{Compliance with ethical standards}

Guarantor The scientific guarantor of this publication is Dr Charlotte Manisty.

Conflict of interest The authors of this manuscript declare no relationships with any companies, whose products or services may be related to the subject matter of the article. 
Statistics and biometry No complex statistical methods were necessary for this paper.

Informed consent No informed consent required.

Ethical approval Institutional Review Board approval was not required because this is a review article.

\section{Methodology}

- observational

- performed at one institution

Open Access This article is distributed under the terms of the Creative Commons Attribution 4.0 International License (http:// creativecommons.org/licenses/by/4.0/), which permits unrestricted use, distribution, and reproduction in any medium, provided you give appropriate credit to the original author(s) and the source, provide a link to the Creative Commons license, and indicate if changes were made.

\section{References}

1. Sutton R, Kanal E, Wilkoff BL et al (2008) Safety of magnetic resonance imaging of patients with a new Medtronic EnRhythm MRI SureScan pacing system: clinical study design. Trials. 9(1):68

2. NHS England Operational Information for Commissioning. Diagnostic imaging dataset. Available via: National Health Service. Available via: https://did.hscic.gov.uk. Accessed 23 June 2017

3. National Institute of Cardiovascular Outcome Research (2016) National Audit of Cardiac Rhythm Management Devices. Available via: http://www.bhrs.com/audit. Accessed 22 June 2017

4. Ahmed HU, El-Shater Bosaily A, Brown LC et al (2017) Diagnostic accuracy of multi-parametric MRI and TRUS biopsy in prostate cancer (PROMIS): a paired validating confirmatory study. Lancet. 389(10071):815-822

5. Bradshaw PJ, Stobie P, Knuiman MW, Briffa TG, Hobbs MST (2014) Trends in the incidence and prevalence of cardiac pacemaker insertions in an ageing population. Open Heart 1(1):e000177

6. Kalin R, Stanton M (2005) Current clinical issues for MRI scanning of pacemaker and defibrillator patients. Pacing Clin Electrophysiol 28(4):326-328

7. Muthalaly RG, Nerlekar N, Ge Y, Kwong RY, Nasis A (2018) MRI in patients with cardiac implantable electronic devices. Radiology. 289(2):281-292

8. Lohrke J, Frenzel T, Endrikat J et al (2016) 25 years of contrastenhanced MRI: developments, current challenges and future perspectives. Adv Ther 33(1):1-28

9. Ainslie M, Miller C, Brown B, Schmitt M (2014) Cardiac MRI of patients with implanted electrical cardiac devices. Heart. 100(5): 363-369

10. Irnich W, Irnich B, Bartsch C, Stermann W, Gufler H, Weiler G (2005) Do we need pacemakers resistant to magnetic resonance imaging? Europace. 7(4):353-365

11. Roguin A, Zviman MM, Meininger GR et al (2004) Modern pacemaker and implantable cardioverter/defibrillator systems can be magnetic resonance imaging safe: in vitro and in vivo assessment of safety and function at $1.5 \mathrm{~T}$. Circulation. 110(5):475-482

12. Indik JH, Gimbel JR, Abe H et al (2017) 2017 HRS expert consensus statement on magnetic resonance imaging and radiation exposure in patients with cardiovascular implantable electronic devices. Heart Rhythm 14(7):e99-e151
13. Lowe MD, Plummer CJ, Manisty CH, Linker NJ (2015) Safe use of MRI in people with cardiac implantable electronic devices. Heart. 101(24):1950-1953

14. Brignole M, Angelo A, Baron-Esquivias G et al (2013) 2013 ESC guidelines on cardiac pacing and cardiac resynchronization therapy. Eur Heart J 34(29):2281-2329

15. Nazarian S, Hansford R, Rahsepar AA et al (2017) Safety of magnetic resonance imaging in patients with cardiac devices. N Engl J Med 377(26):2555-2564

16. Russo RJ, Costa HS, Silva PD et al (2017) Assessing the risks associated with MRI in patients with a pacemaker or defibrillator. N Engl J Med 376(8):755-764

17. Kabil J, Belguerras L, Trattnig S, Pasquier C, Felblinger J, Missoffe A (2016) A review of numerical simulation and analytical modeling for medical devices safety in MRI. Yearb Med Inform 33:152-158

18. Shah AD, Morris M, Hirsh D et al (2018) Magnetic resonance imaging safety in nonconditional pacemaker and defibrillator recipients: a meta-analysis and systematic review. Heart Rhythm 15(7): 1001-1008

19. Wazni O, Epstein LM, Carrillo RG et al (2010) Lead extraction in the contemporary setting: the LExICon study: an observational retrospective study of consecutive laser lead extractions. J Am Coll Cardiol 55(6):579-586

20. Jones SO IV, Eckart RE, Albert CM, Epstein LM (2008) Large, single-center, single-operator experience with transvenous lead extraction: outcomes and changing indications. Heart Rhythm 5(4): $520-525$

21. Poole JE, Gleva MJ, Mela T et al (2010) Complication rates associated with pacemaker or implantable cardioverter-defibrillator generator replacements and upgrade procedures: results from the REPLACE registry. Circulation. 122(16):1553-1561

22. Cruypeninck Y, Dubourg B, Michelin P et al (2017) Pacemakers and MRI: a protocol in line with international guidelines and approved by the SFICV (French Society of Cardiovascular Imaging). Diagn Interv Imaging 98(3):203-215

23. Maglia G, Curnis A, Brieda M et al (2015) Assessing access to MRI of patients with magnetic resonance-conditional pacemaker and implantable cardioverter defibrillator systems. J Cardiovasc Med (Hagerstown) 16(10):715

24. Truven Health (2015) Commercial and Medicare database analysis. Available via: http://www.medtronic.com/us-en/healthcareprofessionals/mri-surescan/implantable-cardiac-devices.html. Accessed 23 June 2017

25. Nazarian S, Reynolds MR, Ryan MP, Wolff SD, Mollenkopf SA, Turakhia MP (2016) Utilization and likelihood of radiologic diagnostic imaging in patients with implantable cardiac defibrillators. $\mathrm{J}$ Magn Reson Imaging 43(1):115-127

26. Sabzevari K, Oldman J, Herrey AS, Moon JC, Kydd AC, Manisty C (2017) Provision of magnetic resonance imaging for patients with "MR-conditional" cardiac implantable electronic devices: An unmet clinical need. Europace. 19(3):425-431

27. Murray AS, Gilligan PJ, Bisset JM, Nolan C, Galvin JM, Murray JG (2018) Provision of MR imaging for patients with cardiac implantable electronic devices (CIEDs): a single-center experience and national survey. Ir J Med Sci. https://doi.org/10.1007/s11845018-1922-y

28. Celentano E, Caccavo V, Santamaria M et al (2017) Access to magnetic resonance imaging of patients with magnetic resonanceconditional pacemaker and implantable cardioverter defibrillator systems: results from the Really ProMRI study. Europace 20(6): 1001-1009

29. Bhuva AN, Feuchter P, Hawkins A et al (2019) MRI for patients with cardiac implantable electronic devices-simplifying complexity with a 'one-stop' service model. BMJ Qual Saf 0:1-6 
30. Jung W, Zvereva V, Hajredini B, Jäckle S (2011) Initial experience with magnetic resonance imaging-safe pacemakers : a review. J Interv Card Electrophysiol 32(3):213-219

31. Padmanabhan D, Kella DK, Mehta R et al (2017) Safety of magnetic resonance imaging in patients with legacy pacemakers and defibrillators and abandoned leads. Heart Rhythm 15(2):228-233

32. Shulman RM, Hunt B (2018) Cardiac implanted electronic devices and MRI safety in 2018 - the state of play. Eur Radiol 28(10): 4062-4065

33. Bhuva AN, Kellman P, Graham A et al (2019) Clinical impact of cardiovascular magnetic resonance with optimized myocardial scar detection in patients with cardiac implantable devices. Int J Cardiol 279:72-78

34. Rashid S, Rapacchi S, Vaseghi M et al (2014) Improved late gadolinium enhancement MR imaging for patients with implanted cardiac devices. Radiology. 270(1):269-274

35. Hilbert S, Jahnke C, Loebe $\mathrm{S}$ et al (2017) Cardiovascular magnetic resonance imaging in patients with cardiac implantable electronic devices: a device-dependent imaging strategy for improved image quality. Eur Heart J Cardiovasc Imaging 19(9):1051-1061

36. Nazarian S, Roguin A, Zviman MM et al (2006) Clinical utility and safety of a protocol for noncardiac and cardiac magnetic resonance imaging of patients with permanent pacemakers and implantablecardioverter defibrillators at 1.5 tesla. Circulation. 114(12):12771284

37. Samar H, Yamrozik JA, Williams RB et al (2017) Diagnostic value of MRI in patients with implanted pacemakers and implantable cardioverter-defibrillators across a cross population: does the benefit justify the risk? A proof of concept study. JACC Clin Electrophysiol 3(9):991-1002

38. Cancer Research UK (2014) Saving lives, averting costs. Available via: https://www.cancerresearchuk.org/sites/default/files/saving lives_averting_costs.pdf. Accessed 24 Sept 2017

39. National Institute for Health and Care Excellence (2015) Transition between inpatient hospital settings and community or care home settings. Enhanced Tariff option; 2015. Available via: https://www. nice.org.uk/guidance/ng27. Accessed 22 June 2017

40. Royal College of General Practitioners, Society and College of Radiographers, The Royal College of Radiologists (2013) Quality imaging services for primary care: a good practice guide. Available via: https://www.rcr.ac.uk/clinical-radiology/publications-andstandards. Accessed 13 Jul 2017.

41. Deloitte Centre for Health Solutions (2013) Working differently to provide early diagnosis: improving access to diagnostics. Available via http://www.deloitte.com. Accessed 22 June 2017

42. Savouré A, Mechulan A, Burban M, Olivier A, Lazarus A (2015) The Kora pacemaker is safe and effective for magnetic resonance imaging. Clin Med Insights Cardiol 9:85-90

43. Philips FieldStrength (2015) Scanning patients with MR Conditional implants. Accessed at: www.philips.co.uk/healthcare/ education-resources/publications/fieldstrength/mri-and-mrconditional-implants. Accessed 5 Oct 2017

44. Center for Medicaid and Medicare Services (2018) Coverage with evidence development. Available via: https://www.cms.gov/ Medicare/Coverage/Coverage-with-Evidence-Development/ MRA_MRI.html. Accessed Feb 2018

45. Kmietowicz Z (2018) Patients with cardiac devices should not be excluded from MRI scans, say experts. BMJ. 362:k3623

46. Sommer T, Bauer W, Fischbach K et al (2017) MR imaging in patients with cardiac pacemakers and implantable cardioverter defibrillators. Rofo 189(3):204-217. https://doi.org/10.1055/s-0043102029

47. British Heart Rhythm Society (2018) Standards for implantation and follow-up of cardiac rhythm management devices in adults. Available at http://www.bhrs.com/standards. Accessed Jan 2018

Publisher's note Springer Nature remains neutral with regard to jurisdictional claims in published maps and institutional affiliations. 